\title{
Retour sur « Le savoir-prendre »
}

\section{Christian Bessy et Francis Chateauraynaud}

\section{OpenEdition}

\section{Journals}

Édition électronique

URL : https://journals.openedition.org/tc/5150

DOI : $10.4000 /$ tc. 5150

ISSN : 1952-420X

Éditeur

Éditions de l'EHESS

\section{Édition imprimée}

Date de publication : 30 juin 2010

Pagination : 686-688

ISSN : 0248-6016

\section{Référence électronique}

Christian Bessy et Francis Chateauraynaud, « Retour sur « Le savoir-prendre »», Techniques \& Culture [En ligne], 54-55 | 2010, mis en ligne le 30 juin 2013, consulté le 29 septembre 2022. URL : http:// journals.openedition.org/tc/5150 ; DOI : https://doi.org/10.4000/tc.5150 


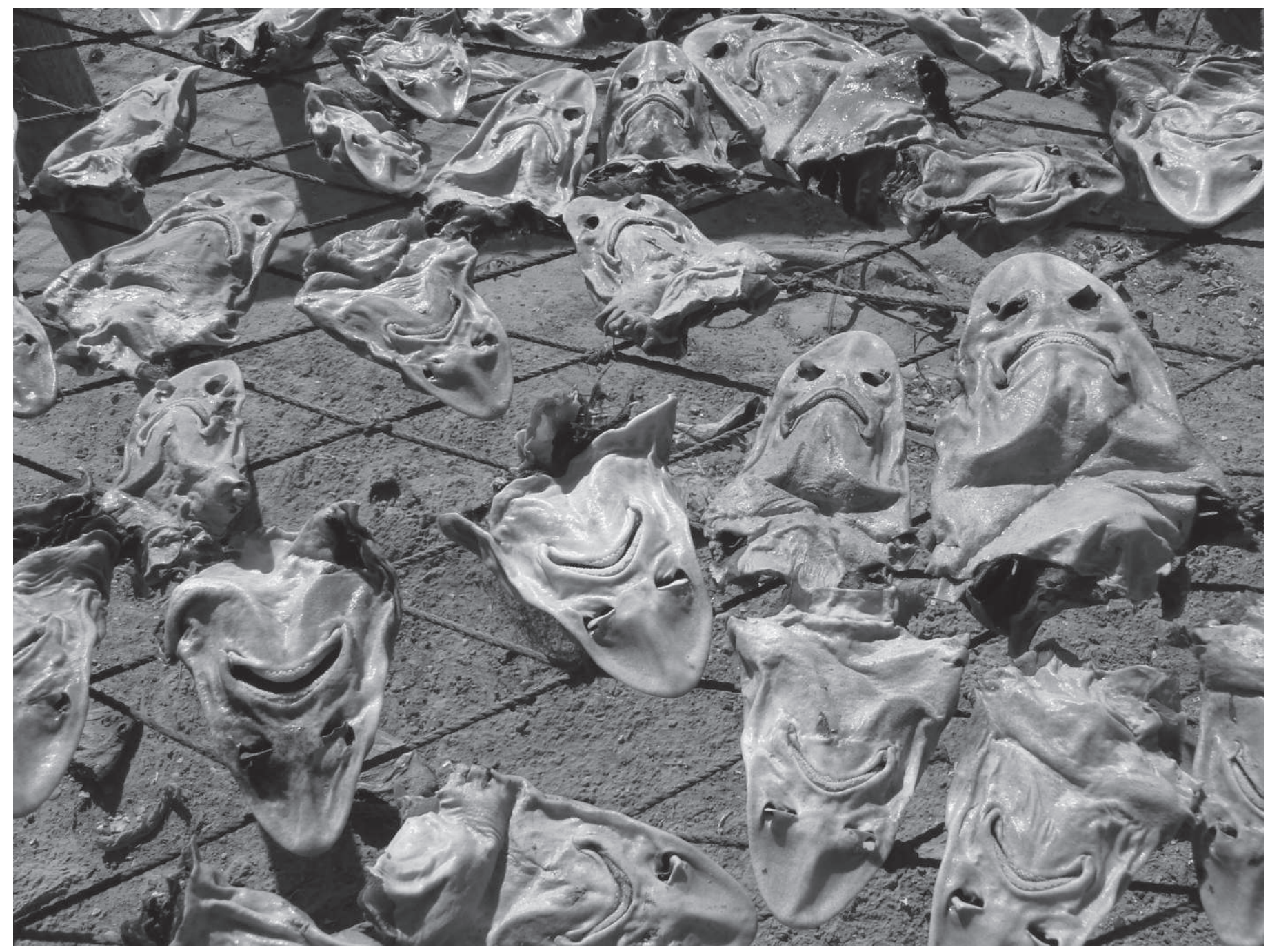




\section{Christian Bessy \& Francis Chateauraynaud}

CNRS/IDHE ENS-Cachan, EHESS

bessy@idhe.ens-cachan.fr

chateau@msh-paris.fr
Cultures matérielles 2 - VIII

Techniques \& Culture 54-55 volume 2, 2010 : 686-688

\section{Retour sur}

\section{"Le savoir-prendre »}

C'est dans le cadre d'une recherche théorique, menée aux points de jonction de la sociologie du jugement et de l'économie des conventions, que l'observation de l'activité des commissaires-priseurs s'est imposée comme un point d'entrée heuristique pour saisir les opérations pratiques qui sous-tendent la qualification des objets dans la vie quotidienne. En renouant avec la démarche ethnométhodologique, nous cherchions en effet à caractériser les processus par lesquels la mise en présence des personnes et des choses conduit à des problèmes d'identification, de caractérisation ou d'évaluation. Pour décrire les procédures de résolution des troubles et les formes d'arbitrage entre les jeux de qualifications disponibles, la notion de « prise » s'est rapidement imposée, au point de former le cœur d'un édifice conceptuel, Experts et faussaires (1995), dont l'article de Techniques et Culture fournit une première esquisse développée à l'issue d'un long parcours d'enquête assez peu conventionnel du point de vue des normes disciplinaires de l'époque. Suivre, dans une recherche, des amateurs d'art, des douaniers, des antiquaires, des experts en contrefaçons, des conseils en brevets ou des avocats en droits d'auteur, des archéologues ou des philologues, des experts judiciaires, des chasseurs de « hoaxes» ou de fraudes scientifiques, des œenologues ou des collectionneurs de voitures anciennes n'allait pas vraiment de soi. Certes, on conçoit aisément après coup que la question de l'authentification qui était au cœur de notre problématique ouvrait sur un champ indéfini d'activités et qu'elle supposait de se déplacer dans l'espace et le temps. Plus qu'une collection d'anecdotes, il nous fallait bâtir un espace de variation. L'exploration systématique des propriétés révélées par cet espace a ainsi permis d'élaborer une théorie de l'expertise alternative, fondée sur une double irréduction: le refus de rabattre les opérations techniques liées aux choses mêmes sur une logique de catégorisation sociale - ce qui est le cas lorsque l'expertise est conçue uniquement comme un acte de certification; le souci 
de ne pas écraser la perception dans le monde sensible sous l'empire du jugement et son expression publique. Clairement distingués par l'analyse détaillée de multiples figures de troubles et de défaillances, les plans peuvent être ensuite réarticulés à l'aide de la notion de prise, qui joue à la fois un rôle analytique et synthétique.

L'émergence de la « théorie de la prise » comme l'ont désignée plus tard des commentateurs bienveillants, s'est produite dans une période d'intenses discussions entre l'économie des conventions et l'anthropologie des sciences et des techniques. En effet, une opposition se creusait entre, d'un côté, l'idée que l'essentiel de la vie sociale est consacré à la fabrication d'accords et de dispositifs de coordination, et, de l'autre, dans le courant connu aujourd'hui sous l'appellation de «théorie de l'acteur-réseau », la mise en avant d'une prolifération d'agencements fondés sur une logique de réseaux engendrant autant de points de passage obligés que de déplacements et d'innovations. En examinant ce qui faisait défaut à ces deux paradigmes, et en explorant les passerelles potentielles entre phénoménologie et sciences cognitives, les enquêtes sur les prises ont contribué à ouvrir une troisième voie. L'enjeu était de prendre au sérieux les expériences sensibles et la complexité des liens qu'elles entretiennent avec les formes d'expression et de jugement, notamment via l'usage des instruments conçu comme une modalisation de corps à corps. L'ethnographie des séances d'estimation et de préparation des ventes publiques menées par des commisseurs-priseurs a, de ce point de vue, joué un rôle décisif. Ce n'est bien sûr pas un hasard si le mot " prise », couramment utilisé sans être thématisé, fait écho à la "prisée », au fait d'« accorder un prix aux choses », ce qui évoque un double processus d'attention et de valorisation des objets.

En inaugurant une voie « réaliste » au cœur des sociologies pragmatiques, l'étude sur l'évaluation des objets a constitué la première mise à l'épreuve d'un renouveau théorique, qui a engendré par la suite de multiples programmes de recherches. Du côté de la sociologie, après une application aux activités pratiques liées aux dispositifs technologiques, comme le pilotage de centrale nucléaire (Chateauraynaud 1997), c'est surtout la « perte de prise » qui a donné lieu aux développements les plus conséquents (Chateauraynaud $\&$ Torny 1999). Explorant une autre dimension de la prise, l'attention à la production des asymétries ou des relations d'emprise, a permis de réinterroger les notions de « domination » et de «pouvoir» et de saisir les techniques utilisées pour « avoir prise sans donner prise », techniques assez subtiles dans un monde en réseau (Chateauraynaud 2006). En économie, outre de multiples applications du côté de l'« économie des singularités » (Karpik 2007) c'est surtout la question des processus d'apprentissage, du rôle des capacités d'arrière-plan et des savoirs pratiques dans la production de la «rationalité », individuelle ou collective, qui a formé l'angle principal de développement de la prise (Bessy 2003). Plus généralement, l'entrée par les prises sur les objets conduit à prendre en compte tous les intermédiaires qui participent à la construction des marchés: tantôt médiateurs, experts ou prescripteurs, ils peuvent aussi exploiter les imperfections informationnelles de façon stratégique (Bessy \& Eymard-Duvernay 1997). 